\title{
Expression and cytokine secretion in the states of immune reactivation in leprosy
}

E.P. Sampaio and E.N. Sarno

\author{
Laboratório de Hanseníase, Instituto Oswaldo Cruz, FIOCRUZ, \\ Rio de Janeiro, RJ, Brasil
}

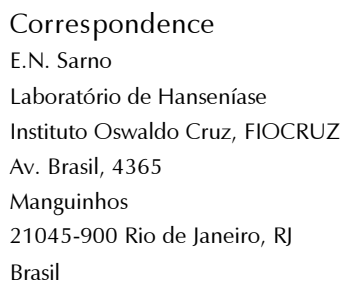

Presented at the International Meeting on Cytokines, Angra dos Reis, RJ, Brasil, November 24-28, 1996.

Received September 4, 1997 Accepted October 13, 1997

\begin{abstract}
Leprosy is a chronic inflammatory disease caused by Mycobacterium leprae. The human response to this pathogen exhibits intriguing aspects $w$ hich are up to now not $w$ ell understood. The present study discusses the probable mechanisms involved in $T$ cell-specific unresponsiveness observed in lepromatous patients. $A$ nalysis of the cytokine profile either in blood leukocytes or in skin specimens taken from leprosy lesions indicates that some parameters of $T \mathrm{~h} l$ immune response are present in lepromatous patients under reactional states.
\end{abstract}

Key words - Leprosy - Reactions - Cytokines

\section{Introduction}

Leprosy is a chronic inflammatory disease caused by Mycobacterium leprae, an obligate intracellular pathogen that provides a model to address the role of $T$ cell subsets in human disease. The outcome of any infection relies on a complex cascade of interactions in vivo. The dynamic nature of the immune response to $M$. leprae is reflected in both the variety of outcomes in different subjects and the $v$ arying responses $w$ ith time by one individual.

Subclinical infection $w$ ith $M$. leprae occurs in a large proportion of those $w$ ho are exposed to the leprosy bacillus. Nevertheless, it is estimated that 10 to 12 million people suffer from leprosy $w$ orld $w$ ide. The disease presents a broad spectrum of clinical forms $w$ hich depend essentially on host immune response. $A \mathrm{t}$ one end of the spectrum lie tuberculoid patients, $w$ ho have localized skin lesions, measurable $T$ cell responsiveness in vitro or in vivo, a strong delayed-type hypersensitivity response, and low levels of serum antibodies to $M$. leprae. $A \mathrm{t}$ the other end of the spectrum are lepromatous patients $w$ ith multiple and diffuse lesions and a diminished skin test response to lepromin, $w$ ho are unable to limit the spread of the bacteria $w$ hich, in the absence of chemotherapy, may disseminate throughout the body. These patients show little detectable in vitro proliferative or in vivo skin test responses specific for $M$. leprae antigens, although serum antibody levels specific for $M$. leprae are very high. In addition, histopathological aspects of leprosy lesions are also characteristic of patients in each group at one pole of the spectrum. Tuberculoid lesions present $w$ ellformed epithelioid granulomas consisting of $T$ lymphocytes and differentiated macrophages. Bacilli are rarely found. Lepromatous lesions consist of macrophages containing 
large numbers of bacteria. Numerous histochemical studies have confirmed the predominance of $C D 4+$ over $C D 8+T$ cells in tuberculoid leprosy, $w$ hile in lepromatous leprosy lesions there are far fewer lymphocytes and similar proportions of $\mathrm{CD} 4+$ and $C D 8+T$ cells (1).

\section{Cellular immune response in leprosy}

Lepromatous leprosy patients display a selective immunological unresponsiveness to Mycobacterium leprae antigen $w$ ith absence of delayed-type hypersensitivity, $T$ cell proliferation, and deficiency in the production of growth factors such as $I L-2$. These patients also fail to produce interferongamma $(I F N \gamma)$ in response to $M$. leprae, $w$ hich seems to correlate $w$ ith the inability of their macrophages to prevent the growth of the mycobacterium. Cellular anergy observed in lepromatous patients appears to be $M$. leprae specific since the immune response against other antigens is largely normal. $A \mathrm{c}$ tive suppression by macrophages and/or $T$ cells may explain their inability to respond to leprosy bacilli. Lepromatous patients carry a high load of bacilli $w$ hich may play a role in the in vivo induction of immune tolerance.

$G$ enetic factors have been considered for a long time in leprosy. This is largely due to the observation of clustering of leprosy around certain families, and the failure to understand $w$ hy certain individuals develop lepromatous leprosy while others develop the non-lepromatous form. $D$ e $V$ ries et al. (2) were the first to find that $H \mathrm{~L} A$ genes are important genetic factors in leprosy. It is evident now that these $H L A$ genes do not determine susceptibility to leprosy per se but rather control the type of leprosy that develops upon infection of susceptible individuals. Interestingly, among the susceptible individuals, those with $H \mathrm{~L} A-D R 3$ more often develop tuberculoid leprosy, $w$ hereas those $w$ ith another $H L A$ type, $D Q 1$, more often develop lepromatous leprosy (2).
The crucial role of $C D 4+T$ helper cells in orchestrating cell-mediated immunity has led to the concept that macrophage activation by cytokines secreted from major histocompatibility complex (MHC) class II-restricted, antigen-specific $T$ helper type $I(T \mathrm{~h} 1)$ cells is the principal mechanism of protection against intracellular microbes. In recent years, however, this concept has undergone major revisions given the important contribution of $C D 8+T$ cells to acquired resistance to at least some mycobacterial species, and the role of $\gamma \delta-T$ cell receptor (TCR) cells in antimycobacterial immunity (3). More recently, novel classes of $T$ cell receptor ligands and antigen presentation molecules have entered the scene. $T$ cells can also recognize nonpeptide ligands such as mycobacterial mycolic acids, lipoarabinomannan and isopentenyl phosphate groups (4). Moreover, peptides as $w$ ell as non-peptide ligands can be presented by non- $M H C$-encoded but $M H C$ like molecules such as $C D 1$. It is completely unclear, however, to $w$ hat extent these novel $T$ cell receptor ligands precisely contribute to protective immunity or immunopathology, and whether the results obtained in experimental infections in mice can be extrapolated to naturally occurring chronic infection in humans.

Moreover, recent studies have focused on the cytokine profiles of $M$. leprae reactive $T$ cells. It has been proposed that the two polar stable forms of leprosy reflect the dominance of two major $T$ cell subpopulations. The hypothesis that the spectrum of leprosy reflects the balance between $T$ helper ( $T \mathrm{~h} I$ and $T \mathrm{~h} 2$ ) cell populations activated by mycobacteria, defining the cytokine profile produced by each of these populations (5), is indeed exciting, but the data accumulated so far have not backed it up. In tuberculoid leprosy, $T \mathrm{~h} /$ cells producing both $\mathrm{IL}-2$ and $I F N \gamma$ may lead to an efficient immune response $w$ hile in lepromatous leprosy, the $T h 2$ population may be preferentially acti$v$ ated, producing both $I L-4$ and $I L-5$. Studies 
have provided evidence in favor of this hypothesis, $w$ hereas others have provided contrasting results.

Instability in the immune response to M. leprae

$A$ ctually, leprosy is not a stable disease and the above described polar forms of leprosy are rarely diagnosed at $C$ linical Units. $B$ etween these two extremes (polar forms) a continuous clinical and histopathological range, termed borderline leprosy, is more often seen. In this case, the cell-mediated immune response is not stable and patients present an increased immunological response to $M$. leprae as they approach the tuberculoid end of the spectrum.

Interestingly, we have recently demonstrated that biopsies from some unreactional borderline lepromatous patients obtained at diagnosis show the pattern of in situ activation instead of the histological characteristics of non-responders. Presence of high numbers of $C D 4+T$ cells in the inflammatory infiltrate, thickening of the epidermis, and expression of $H \mathrm{~L} A-D R$ and intracellular adhesion molecule (ICAM-1) on the surface of keratinocytes have been noted (6).

$A$ t this point, the following questions demand clarification: 1) May the pattern of immune response seen in borderline patients be the same as found in the polar forms of leprosy? 2) Is the non-responsiveness seen in borderline lepromatous patients the result of a predominance of a $T \mathrm{~h} 2$ over a $T \mathrm{~h} 1$ response? 3) May the cytokine profile expressed by these patients be the result of a $T h 0$ response? 4) May the non-responsiveness seen in these patients be modulated by the presence of mycobacterium components, such as LAM and PGL 1 , known to have suppressor properties on $T$ cells and macrophages?

In a very recent study (7), the functional properties of $C D 4+C D 8-T$ cell clones isolated from the skin lesions and blood of leprosy patients across the spectrum were assayed. Substantial heterogeneity in the production of cytokines $w$ as observed. $A$ variety of patterns of cytokine secretion distinct from those of $T \mathrm{~h} 1, T \mathrm{~h} 2$ or $T \mathrm{~h} 0 \mathrm{w}$ as evident, although no striking correlation $w$ ith clinical status $w$ as apparent. Noteworthy $w$ as the large number of clones from skin which secreted neither $I \mathrm{~L}-2$ nor $I \mathrm{~L}-4$, but large amounts of tumor necrosis factor alpha $(T N F \alpha)$ and $I F N \gamma$.

In another study, following the analysis of cytokine $\mathrm{m} R N A$ expression by $R T-P C R$ and cytokine secretion in in vitro stimulated peripheral blood mononuclear cells (PBMC) of leprosy patients, it $w$ as found that approximately $50 \%$ of all individuals showed a Th0-like mixed cytokine pattern, irrespective of the clinical status of leprosy or the antigen used (8).

$A$ dditional data from many laboratories have made important and also controversial contributions: i) addition of $I \mathrm{~L}-2$ to patient cultures restored the $M$. leprae unresponsiveness in some lepromatous patients (9); ii) addition of $I L-4$ to the cultures increased the pre-existing $M$. leprae response; iii) preincubation of patient cultures $w$ ithout any stimulus reversed the specific $T$ cell unresponsiveness of lepromatous patients (10); iv) unresponsiveness to $M$. leprae could also be overcome in vitro by stimulation $w$ ith $M$. leprae components in some patients.

In vivo immune reactivation in leprosy has also been demonstrated, as follows: i) changes in $T$ cell populations $w$ ere produced following $P P D, \mathrm{r} I F N \gamma$ and $\mathrm{r} / \mathrm{L}-2$ injection, thereby eliciting upgrading aspects in the lepromatous lesions $w$ ith increasing numbers of $C D 4+$ cells (11-13); ii) upgrading of lepromatous patients submitted to repeated vaccination $(B C G, M$. leprae, Mycobacterium w) (14); iii) presence of $M$. lepraereactive $T$ cells in lepromatous patients during the reactional episodes $(15,16)$; iv) parameters of $T$ cell activation have been observed in biopsies obtained from leproma- 
tous patients during reaction; v) a marked influence on the immunological response to $M$. leprae (a positive response) $w$ as observed in some lepromatous leprosy patients submitted to specific chemotherapy (17).

Immunological reactivity and cytokine production during reactions in leprosy

During the course of leprosy, a reasonable proportion of patients will develop reactional episodes of acute inflammation affecting the skin and nerves. Leprosy reactions provide a window through $w$ hich one can observe the dynamics of the immune response to $M$. leprae.

These reactional states are classified as type $I(R R)$ or type $I I(E N \mathrm{~L})$ reaction depending on the clinical characteristics of the acute episode and its immune background. $B$ oth the precipitating factors and the physiopathological mechanisms involved in reactions remain ill defined. $A$ lthough the outcome of this reactivation seems to be the elimination of bacilli or their constituents, irreversible peripheral nerve damage may occur in a small number of patients. The ultimate concern in leprosy must be the identification of inflammatory cytokines involved in the induction of tissue damage. Evidence implicating $T N F \alpha$ as a pivotal molecule in this process exists. $T N F \alpha$ seems to be a natural anti-infectious agent involved in bacterial resistance, $w$ hich circumstantially pro$v$ okes side effects at the site $w$ here elevated concentration is reached.

The acute episodes of immunological and inflammatory responses are associated $w$ ith changes in immunological reactivity $w$ hich seem to occur in both types of reaction. Tissue damage that occurs during the reactional states could be the result of opportunities generated during the evolution of $M$. leprae infection involving specific and nonspecific cell-mediated immunity. The fragile equilibrium between activation and inhibi- tory molecules in the circulation and binding tissue molecules at the site of the skin infection is interrupted, generating a new order between pathogen and host. $T N F \alpha$, a critical molecule related to tissue damage in leprosy, is overproduced during reaction (18), and $I \mathrm{~L}-6, I \mathrm{~L}-8$ and $I F N \gamma$ may be associated secreted factors (19). On the other hand, adhesion molecules constitutively expressed in the tissue or induced during the activation of cell-mediated immune response seem to collaborate, making the infected tissue more susceptible to the toxic effects of $T N F \alpha$. The prompt beneficial effects obtained following treatment of reactional patients $w$ ith thalidomide and/or steroids indicate that these episodes are not due to the aggravation of the infection itself, but are rather the result of the generation and release of excessively high amounts of host immunological mediators.

Histologically, reactional lesions contain a dense cellular infiltrate composed predominantly of lymphocytes (CD4+T cells), monocytes and neutrophils (only seen in type $I I$ reaction). This infiltrate is also accompanied by increases in epidermal thickness, keratinocyte $M H C$, class $I I$ antigen expression, an increased number of epidermal Langerhans ' cells and the presence of intraepithelial $T$ cells in the reactional lesion. Enhanced expression of $I C A M-1$ on the keratinocytes and leukocyte function antigen 1 (LFA-1) has recently been described (19).

One may speculate that reaction occurs in the course of leprosy $w$ hen a patient develops an increased cell-mediated immune response against $M$. leprae and, in some situations, the patient's clinical state could move tow ard the tuberculoid end of the spectrum.

$A$ lthough $v$ ery important conclusions can be drawn from a vertical study, only a sequential analysis of the immunological parameters from each individual patient $w$ ill allow us to understand the actual mechanisms underlying the development of reactions in leprosy. 
In an attempt to overcome part of these problems, $w$ e designed a longitudinal prospective study which would allow us to perform a sequential analysis in some patients. $A$ ccordingly, we started by addressing the following questions: 1) Could cell-mediated immunity arise from a previously unresponsive multibacillary leprosy patient during the reactional episode? 2) $W$ ould this $T$ cellmediated immune response be long lasting, moving the patient toward the tuberculoid end of the spectrum? 3) Would the cytokine profile determine these changes in the immune response? 4) Which cell populations participate in the triggering and regulation of these events?

Patients $w$ ho developed a reaction during multiple drug treatment $w$ ere submitted to immunological evaluation before, during and after the reactional episode and their data $w$ ere described thereafter. Blood and tissue samples $w$ ere collected from all patients during the study. Peripheral mononuclear blood cells $w$ ere obtained by Ficoll-Hypaque density centrifugation. The lymphoproliferation assay, measurement of $I F N \gamma$ in a 5-day culture supernatant and analysis of cytokine $\mathrm{m} R N A$ expression were performed in all patients. Histological and immunohistological analysis $w$ as done in 23 patients (a full description is given below). Serum samples $w$ ere also collected from all patients before, during and after the reaction.

In this preliminary analysis, $66.6 \%$ of the patients tested showed an in vitro response to $M$. leprae during the reactional episode, as analyzed in the lymphoproliferation and $I F N \gamma$ assays. When the immunological proliferative response to $M$. leprae $w$ as tested before and during the reactional episode, multibacillary patients $w$ ho $w$ ere unresponsive to $M$. leprae antigen became good responders during the reaction. More interestingly, the majority of patients who showed this positive immune response during the reaction shifted back to low responder status after the reactional episode subsided. $A$ nother impor- tant observation refers to the patient's response to the lepromin skin test. $A$ lthough most patients became responsive to the skin test during the reaction, about $47.6 \%$ of them still had a positive lepromin response after the reactional episode.

Biopsies from 23 patients (a total of 48 samples) $w$ ere submitted to immunohistochemical staining $w$ ith a large panel of monoclonal antibodies. The number of $\gamma \delta-T C R$ positive cells as well as $C D 1$-positive cells in the dermis $w$ as considerably increased during the reactions and remained relatively high in the post-reactional lesions. Gamma delta- $T$ cells $w$ ere not detected in pre-reactional lesions as opposed to the reactional lesions, $w$ hich reached $v$ ery high numbers in some cases. The $C D 4 / C D 8$ ratio, although slightly $>1$ in pre-reactional lesions (mean $1.23 \pm 0.36)$, $w$ as elevated in reactional lesions $(1.77 \pm 1.41)$ and remained high in post-reactional lesions $(1.97 \pm 1.56)$ as $w$ ell.

There $w$ as an increased influx of $C D 4+T$ cells to the site of reaction. The functional characterization of this $T$ cell subset in each of the reactional states may elucidate some current questions. There is indirect evidence that the $C D 4+T \mathrm{~h} 1$ subset predominates in these lesions since other parameters of local immune reactivation known to be induced by $I F N \gamma$ are presentin situ, such as increased expression of $H \mathrm{~L} A-D R$ as $w$ ell as ICAM-I by $k$ eratinocytes and endothelial cells. Interestingly, these alterations remained high in post-reactional biopsies as $w$ ell. The effect of $I F N \gamma$ and $T N F \alpha$ in inducing keratinocyte membrane activation is very $w$ ell known and may justify the changes detected in the reactional lesions. It is now $w$ idely accepted that overexpression of integrins on the endothelial cell surface is one of the first steps in the immuno-inflammatory cascade. These data indicate that the cell-mediated immune response is present in multibacillary patients during the reaction. The positive response of patients seen in vitro in the lymphoproliferation test and in the IFN $\gamma$ assay supports this 
conclusion. A nother valuable contribution is the demonstration of the decay of cellmediated immunity in the same patients tested at $v$ arious times after reaction. By this time, lymphoproliferation (LTT) and $I F N \gamma$ had returned to initial levels.

It seems that reversal reaction episodes occurring in unresponsive multibacillary patients may represent a unique moment in the course of leprosy infection, in which a break of the anergic state takes place.

$A$ recent report (20) has demonstrated that the clinical course of a lepromatous leprosy patient $w$ as determined by the cytokine profile produced by different subtypes of $C D 4+T$ cells. The predominant $T \mathrm{~h} 2$ profile seen by $R T-P C R$ during the disease $w$ as replaced by the $T \mathrm{~h} 1$ profile on the occasion of reaction. This might indicate that studies on the profiles of cytokines in leprosy may not be reliable if done at only one time during the evolution of the disease. The decision as to $w$ hich cytokine profile $w$ ill be synthesized may lie in the balance between genetic factors and local environmentally acquired factors (IL-2, IFN $\gamma$, adjuvant, LPS, drugs, superantigens, $v$ accination, associated infections and others).

$A$ sequential analysis of cytokine $\mathrm{m} R N A$ expression in the blood and in the lesions of patients from our cohort $w$ as of interest. Cytokine gene expression in PBMC w as assayed directly after isolation from the blood and in the absence of any in vitro stimulation. $S k$ in biopsies $w$ ere also collected and the tissue $w$ as homogenized in a Polytron $P T-3000$ apparatus using Trizol (or RNA zol). Reagent $R N A$ was purified according to manufacturer instructions and $w$ as then $q$ uantitated by absorbance readings at $260 \mathrm{~nm}$. The integrity of RNA samples $w$ as monitored by agarose gel electrophoresis. Good $q$ uality $R N A w$ as obtained and $w$ as available for analysis by $R T-P C R$. A fter reverse transcription, $\mathrm{c} D N A$ was amplified for $P C R$ evaluation in the presence of specific primer pairs for each of the cytokines tested (21).
For semiquantitative $R T-P C R, P C R$ products $w$ ere electrophoresed, transferred to nylon membranes and hybridized to the oligonucleotide probe. $A \mathrm{fter}$ successive $w$ ashings, the filter $w$ as exposed to $X$-ray film for 24 $48 \mathrm{~h}$. Cytokine PCR products $w$ ere quantitated using an analytic imaging system. The same amount of $\mathrm{c} D N A$ was used in each experiment. The results $w$ ere normalized to the amount of actin in each sample, so that $w$ e $w$ ere able to compare distinct intensity of the cytokine bands obtained from the lesions of different samples.

$I \mathrm{~L}-1 \beta, I \mathrm{~L}-6, \mathrm{LL}-8, T N F \alpha, I F N \gamma, G M-C S F$, $I \mathrm{~L}-2 R \mathrm{p} 55$ and perforin gene expression $w$ as detected mainly in reactional patients as compared to the other groups. Most of the unreactional patients were negative for the cytokines tested. None of the cytokine genes $w$ ere detected in normal individuals or in lepromatous patients at the end of multiple drug treatment. In these patients, only actin $\mathrm{m} R N A w$ as detected.

In the present study, it $w$ as possible to detect differences in $T N F \alpha$ gene expression in a lesion from the same patient $w$ hen analyzed before and at the onset of a reactional episode. For the 8 patients tested, the amount of $T N F \alpha \mathrm{m} R N A$ was increased in tissue during reaction, and $w$ as decreased thereafter following treatment. The expression of $I F N \gamma \mathrm{m} R N A$ was detected in the lesion of 4 out of the $6 E N L$ patients tested, whereas 3 out of $3 R R$ patients analyzed were positive for $I F N \gamma$ in situ. Likew ise, $I F N \gamma$ gene expression in the blood and in tissue $w$ as downregulated following treatment for reaction.

In summary, tissue studies also demonstrated that cytokines play a $k$ ey role in all types of reactional lesions. Studies carried out on tissue lesions confirmed that $T N F \alpha$ positive cells (as detected by immunocytochemistry) are a constant finding in reactional biopsies, although some positive cells can also be detected in non-reactional lesions. Moreover, using semiquantitative $R T$ $P C R$ analysis, it $w$ as possible to detect dif- 
ferences in $T N F \alpha$ gene expression in the lesion from the same patient $w$ hen analyzed before and at the onset of a reactional episode. Thus, as demonstrated by kinetic studies, $T N F \alpha$ is undoubtedly increased during reactions in the same individual both in vitro (following stimulation of the cells $w$ ith $T N F \alpha$ agonists) and in vivo (increased protein levels in the serum, and increased cytokine gene expression in situ).

Significant data suggesting that $I F N \gamma$ molecules are in some $w$ ay related to the triggering of $T N F \alpha$ overproduction $w$ ere also obtained. The importance of $I F N \gamma$ in the regulation of cytokine production $w$ as also suggested by the appearance of reaction in patients after intradermal injections of $I F N \gamma$ (22). $W$ e have already demonstrated $I F N \gamma$ priming of blood monocytes for enhanced agonist-induced $T N F \alpha$ release both in vitro and in vivo. Thus, $I F N \gamma$ in the sera of leprosy patients could lead to priming of patient monocytes resulting in enhanced $T N F \alpha$ production and induction of reaction.

$W$ ithin this context, the observation that $P B M C$ obtained from reactional patients released higher $T N F \alpha$ levels than did monocytes obtained from the same individuals $w$ as intriguing. Interestingly, if purified monocytes are reconstituted in vitro $w$ ith lymphocytes, levels of $T N F \alpha$ are similar to those released in the original $P B M C$ cultures from the same individuals. It has recently been pointed out that IFN $\gamma$ might be involved in $T N F \alpha$ overproduction. However, definitive studies showing that $I F N \gamma$ is being synthesized in these cultures and its cell source are still missing.

In the present study, we were able to suggest that 1) the borderline forms of leprosy are in principle clinically and immunologically unstable forms in which reactivation states are most likely to occur. 2) The cytokine profile expressed by these patients either in vivo or in vitro may not be related to any pattern of cytokine secretion already described. 3) The acute reactional episodes that develop in leprosy are associated $w$ ith changes in cellular immune response since parameters of immune reactivation are detected both in vivo and in vitro. 4) The immunological reactivation states seen along the chronic course of leprosy are the result of imbalanced local production and release of immuno-inflammatory cytokines (mainly $I F N \gamma$ and $T N F \alpha$ ) that may induce tissue injury.

\section{References}

1. Britton WJ (1993). Immunology of leprosy. Transactions of the Royal Society of Tropical Medicine and Hygiene, 87: 508514

2. De Vries RR (1991). Genetic control of immunopathology induced by Mycobacterium leprae. American Journal of Tropical Medicine and Hygiene, 44: 12-16.

3. Kaufmann SHE (1993). Immunity to intracellular bacteria. Annual Review of Immunology, 11: 129-163.

4. Sieling PA, Chatterjee D, Porcelli SA, Prigozy TI, Mazzaccaro RJ, Soriano T, Bloom BR, Brenner MB, Kronnenberg M, Brennan PJ \& Modlin RL (1995). CD1 restricted $T$ cell recognition of microbial lipoglycan antigens. Science, 269: 227230.
5. Ramos T, Zalcberg-Quintana I, Appelberg R, Sarno EN \& Silva MT (1989). T-helper cell subpopulations and the immune spectrum of leprosy. International Journal of Leprosy, 57: 73-81.

6. Alvarenga FBF (1995). Cellular immunity in reversal reaction: Immunohistochemical characterization in sequential cutaneous lesions. Doctoral thesis, Universidade Federal Fluminense, Rio de Janeiro.

7. Howe RC, Wondimu A, Demissee A \& Frommel D (1995). Functional heterogeneity among $\mathrm{CD} 4+\mathrm{T}$ cell clones from blood and skin lesions of leprosy patients. Identification of $T$ cell clones distinct from Th10, Th1 and Th2. Immunology, 84: 585594.
8. Mishra N, Murtaza A, Walker B, Narayan NPS, Mishra RS, Ramesh V, Singh S, Colston MJ \& Nath I (1995). Cytokine profile of circulating $T$ cells of leprosy patients reflects both indiscriminate and polarized T-helper subsets: T-helper phenotype is stable and uninfluenced by related antigens of Mycobacterium leprae. Immunology, 86: 97-103.

9. Kaplan G, Weinstein DE, Steinman RM, Levis WR, Elvers V, Patarroyo ME \& Cohn ZA (1985). An analysis of in vitro $T$ cell responsiveness in lepromatous leprosy. Journal of Experimental Medicine, 159: 917-929. 
10. Mohagheghpour N, Gelber RR \& Engleman EG (1987). T cell defect in lepromatous leprosy is reversible in vitro in the absence of exogenous growth factors. Journal of Immunology, 138: 570574.

11. Kaplan G, Nusrat A, Sarno EN, Job CK, McElrath J, Porto JA, Nathan CF \& Cohn ZA (1987). Cellular responses to the intradermal injection of recombinant human $\gamma$ interferon in lepromatous leprosy patients. American Journal of Pathology, 128: 345-353

12. Kaplan G, Sheftel G, Job CK, Mathur NK, Nath I \& Cohn ZA (1988). Efficacy of a cellmediated reaction to the purified protein derivative of tuberculin in the disposal of Mycobacterium leprae from human skin. Immunology, 85: 5210-5214.

13. Kaplan G, Kiessling R, Teklemariam S, Hancock G, Sheftel G, Job CK, Converse P, Ottenhoff THM, Belumink MB, Dietz M \& Cohn ZA (1989). The reconstitution of cell-mediated immunity in the cutaneous lesions of lepromatous leprosy by recombinant interleukin-2. Journal of Experimental Medicine, 169: 893-907.
14. Convit J, Aranzazu N, Ubich M, Pinardi ME, Reyes O \& Alvarado J (1982). Immunotherapy with mixture of Mycobacterium leprae and BCG in different forms of leprosy and in Mitsuda negative contacts. International Journal of Leprosy, 50: 415424.

15. Laal S, Bhutani LK \& Nath I (1985). Natural emergence of antigen-reactive $T$ cells in lepromatous leprosy patients during erythema nodosum leprosum. Infection and Immunity, 50: 887-892.

16. Laal S, Mishra RS \& Nath I (1987). Type I reactions in leprosy: Heterogeneity in Tcell functions related to the background leprosy type. International Journal of Leprosy, 55: 481-487.

17. Esquenazi $D$, Sampaio EP, Moreira AL, Gallo MEN, Almeida SMR \& Sarno EN (1990). Effect of treatment on immune responsiveness in lepromatous leprosy patients. Leprosy Review, 61: 251-257.

18. Sarno EN, Grau GE, Vieira LMM \& Nery JAC (1991). Serum levels of tumour necrosis factor-alpha and interleukin-1 1 during leprosy reactional states. Clinical and Experimental Immunology, 84: 103-108.
19. Sampaio EP, Kaplan G, Miranda A, Nery JAC, Miguel CP, Viana SM \& Sarno EN (1993). The influence of thalidomide on the clinical and immunological manifestation of erythema nodosum leprosum. Journal of Infectious Diseases, 168: 408414.

20. Yamamura $M$, Wang $X$, Ohmen JD, Uyemura K, Rea TH, Bloom BR \& Modlin $R L$ (1992). Cytokine patterns of immunologically mediated tissue damage. Journal of Immunology, 149: 1470-1475.

21. Ehlers S \& Smith KA (1991). Differentiation of $T$ cell lymphokine gene expression: the in vitro acquisition of $\mathrm{T}$ cell memory. Journal of Experimental Medicine, 173: 25-36.

22. Sampaio EP, Moreira AL, Sarno EN, Malta AM \& Kaplan G (1992). Prolonged treatment with recombinant interferon $\gamma$ induces erythema nodosum leprosum in lepromatous leprosy patients. Journal of Experimental Medicine, 175: 1729-1737. 